\title{
Internal Model Control of a Multilink Flexible Manipulator
}

\author{
Waweru Njeri*, Minoru Sasaki and Kojiro Matsushita \\ Department of Mechanical Engineering, Gifu University, 1-1 Yanagido, Gifu, 501-1193, Japan \\ ${ }^{*}$ Corresponding author
}

\begin{abstract}
This paper investigates the use on the internal model control technique in the mitigation of link vibration on a flexible manipulator with load disturbances. Flexible manipulators have gained popularity in the engineering field which require precision at high speed of operation. However, they suffer from link vibrations especially when operated at high speed or when subjected to external disturbances. This limits their application to those where the speed of operation and/or accuracy is not a factor. Researchers have attempted to address this issue using methods such as fixed digital filters, adaptive filters etc. Internal model control technique employs an element of the plant to control it. From the simulation results, we found that IMC is a robust method for the mitigation of link vibration.
\end{abstract}

Keywords-internal model control; flexible manipulator; robust control.

\section{INTRODUCTION}

Flexible manipulators find numerous engineering applications ranging from placement of the read/write head at the right position on a hard disk to fabrication of computer motherboards where electronic components are placed and soldered. Due to their light weight, they are also often used is space exploration. Due to loading and their flexible nature, at high speeds, they tend to vibrate taking a lot of time before they can precisely settle on the desired position [1]. This limitation is exacerbated with increase in the number of links. This is due to the coupled nature of the links.

Over the years, research efforts have been carried out to mitigate this shortcoming. Digital filters, either Finite Impulse(FIR) or Infinite Impulse Response(IIR) were designed to eliminate the dominant strain frequencies in the feedback path. Their main limitation was their fixed frequency response. Changes in the loading or the trajectory may introduce new modes different from the filter cutoff frequencies or shift the dominant frequencies altogether.

Adaptive notch filters have also been used [2] and [3]. In this type of solution, the limitation of fixed response was addressed. These filters adapt the frequency response to the existing modes and any other modes that may arise. The challenge with the notch filter is that the bandwidth was not wide enough to capture all strain modes. This way, some frequencies remain in the system. Also, the notch cannot be shifted over infinite frequencies and adaptive filters only have finite range of operation. Thus adaptation can only be achieved within a certain range of frequencies.
Researchers in [4] and [5] proposed an adaptive direct strain gain by tuning to the measured strain. In their research, they developed an adaptive feedback strain control law by varying the feedback gain for the various loadings. In addition to the vibration mitigation, none of these methods was capable of rejecting disturbances such as jerks etc.

Mahamood in [6] proposed the direct hybrid control based technique to automatically tune the gains of a hybrid Proportional Integral Derivative online. In this scheme, the P, I and $\mathrm{D}$ gains were tuned in an adaptive manner based on the variations in the payload, control torque and the desired trajectories.

To this end, we have developed a robust internal model control for a two-link 3D flexible manipulator for the mitigation of link vibration. Simulations were carried out in Matlab considering load disturbances in addition to the reference joint angles.

\section{INTERNAL MODEL CONTROLLER}

Internal Model control(IMC) is a robust control technique which relies on the internal model of the plant to accurately control it. Developed in the late eighties by Morari and Zafiriou[7],[8], IMC has the strength of simple structure, robust and accurate tracking.

\section{A. Mathematical developemt of IMC}

The internal model control (IMC), has been widely used in the fields of control of chemical processes, is a novel control method based on the process mathematical model. It has the features of a simple structure, accurate tracking ability and strong robustness [9]. Fig 1(a) shows the structure of IMC, where $\mathrm{R}(\mathrm{s}), \mathrm{Y}(\mathrm{s})$ are the input and the output signal of a system, respectively; $\mathrm{Q}(\mathrm{s})$ is the controller, $\mathrm{C}(\mathrm{s})$ denotes the internal model controller; $\mathrm{P}(\mathrm{s})$ is the plant to be controlled; $\mathrm{M}(\mathrm{s})$ means the internal model of the plant; $\mathrm{d}(\mathrm{s})$ is the disturbance; and $\tilde{d}(s)$ represents the error between the system output $\mathrm{Y}(\mathrm{s})$ and the output of the internal $\tilde{Y}(s)$. 


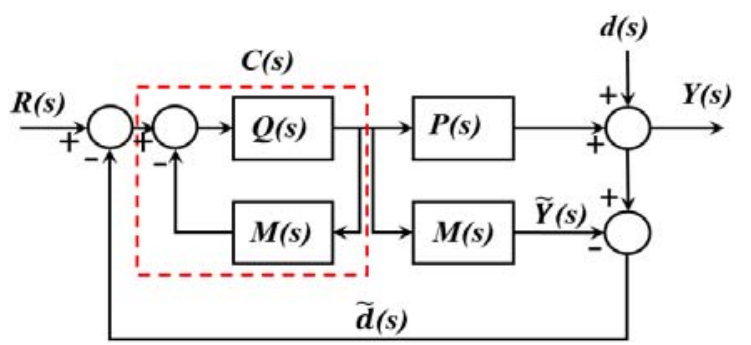

(a)

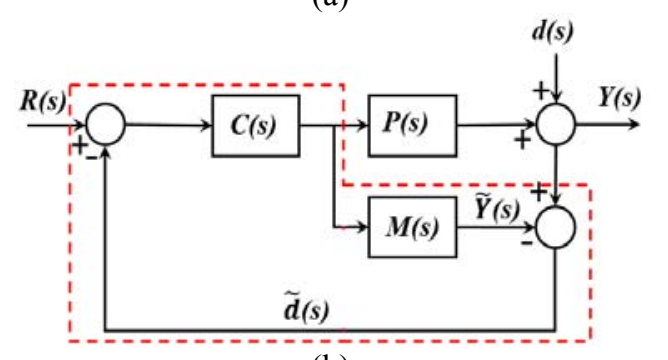

(b)

FIGURE I. IMC ARCHITECTURE

IMC is a control method based on the classical feedback control. The internal model controller $\mathrm{C}(\mathrm{s})$ can be constituted by introducing the internal model of plant $\mathrm{M}(\mathrm{s})$, where $\mathrm{Q}(\mathrm{s})$ and $\mathrm{M}(\mathrm{s})$ are designed as a feedback controller $\mathrm{C}(\mathrm{s})$, as shown in Fig. 1(b), i.e.,

$$
C(s)=\frac{Q(s)}{1+Q(s) M(s)}
$$

Which is equivalent to

$$
\mathrm{Q}(\mathrm{s})=\frac{\mathrm{C}(\mathrm{s})}{1-\mathrm{C}(\mathrm{s}) \mathrm{M}(\mathrm{s})}
$$

Using block reduction formulas, the output $\mathrm{Y}(\mathrm{s})$ can be expressed as

$$
Y(s)=\frac{C(s) P(s) R(s)+[1-C(s) M(s)] d(s)}{1+C(s)[P(s)-M(s)]}
$$

The equation above can further be rewritten as

$$
Y(s)=\tau R(s)+s d(s)
$$

Where

$$
\tau=\frac{C(s) P(s)}{1+C(s)[P(s)-M(s)]}
$$

and

$$
s=\frac{1-C(s) M(s)}{1+C(s)[P(s)-M(s)]}
$$

are the sensitivity and the complementary sensitivity functions of the system respectively. Further, $\tau+s=1$.

It is obvious from the above equation that when $\mathrm{P}(\mathrm{s})=$ $\mathrm{M}(\mathrm{s})$, if $(1-\mathrm{C}(\mathrm{s}) \mathrm{M}(\mathrm{s}))=0$, that is, $\mathrm{C}(\mathrm{s})=\mathrm{M}^{-1}(\mathrm{~s})$, the model has a perfect match with the control objective. Under this condition, $\mathrm{Y}(\mathrm{s})=\mathrm{R}(\mathrm{s})$, which means that the system output is only equal to the input, without any influence by the disturbance. This results in a strong robustness [9]. However, this matching system is quite sensitive to the model deviations, making it hard to be realized in practice. In this case, the internal model controller is generally designed by using an ideal controller, and augmented with a lowpass filter as the correction circuit to improve on the dynamic performance and robustness of system.

From Fig. 1(a), it can be observed that the IMC has the following characteristics

i. The output deviation caused by disturbance $d(s)$ can be reduced. Assume a positive disturbance d(s) that will lead to an increment in the control output $\mathrm{Y}(\mathrm{s})$, this will makes the error $\tilde{d}(s)=\mathrm{Y}(\mathrm{s})-\tilde{Y}(s)$ increase and the input error $\mathrm{R}(\mathrm{s})-\tilde{d}(s)$ will decrease. Following this, the IMC output $\mathrm{Y}(\mathrm{s})$ is decreased, and the output value of controller is decreased. This way, the effect of disturbance influence has been contained effectively.

ii. The output error caused by the error between the plant and its model, i.e. M(s)- P(s), can be reduced. When $\mathrm{M}(\mathrm{s})>\mathrm{P}(\mathrm{s})$, the system output $\mathrm{Y}(\mathrm{s})$ is greater than the IMC output $\tilde{Y}(s)$ making the error $\tilde{d}(s)=\mathrm{Y}(\mathrm{s})-\tilde{Y}(s)$ decrease and the input error R(s)-d(s) increase. Following this, the error $\mathrm{d}(\mathrm{s})$ increases and the input error $[\mathrm{R}(\mathrm{s})-\tilde{d}(s)]$ is decrease, thus the system output is decreased, which means the IMC can reduce the error caused by the model inaccuracy.

iii. When the plant and its model are identical, i.e., M(s) $=\mathrm{P}(\mathrm{s})$ and $\mathrm{C}(\mathrm{s})=\mathrm{G}^{-1}(\mathrm{~s})$, any disturbance $\mathrm{d}(\mathrm{s})$ can be restrained. Thus, the zero-error tracking of input $\mathrm{R}(\mathrm{s})$ can be realized. In this case, the sensitivity of the system is equal to unity as the complementary sensitivity goes to zero.

\section{B. Plant Inversion}

In subsection $A$, we have demonstrated how the disturbance can be rejected by choosing the controller to be the inverse of the plant model. Conventionally, researchers have been inverting the transfer matrix of the plant to get the inverse. In this paper, a different approach is employed. Inversion is achieved by isolating the internal dynamics from the output variable before solving and stabilizing the internal dynamics.

Consider the minimal state space realization of the plant given by

$$
\begin{aligned}
& \dot{x}(t)=A x(t)+B u(t) \\
& y(t)=C x(t)
\end{aligned}
$$

Where $\mathrm{x}(\mathrm{t})$ is the state vector, $u(t)$ is the input and $y(t)$ is the output. To find the input $u_{f f}$ that exactly tracks the desired output $y_{d}(t)$ of the system, we differentiate the output until the input appears explicitly in the expression. Hence

$$
y^{(r)}(t)=C A(r) x(t)+C A(r-1) B u(t)
$$


Where $r$ is the relative degree of the system and superscript (r) denote the rth time derivative. From (1) the inverse feedforward input $u_{f f}(t)$ which tracks the desired trajectory $y_{d}(t)$ can be obtained directly as

$$
u_{f f}(t)=\left(C A^{(r-1)}\right)^{-1}\left[y_{d}^{(r)}-C A^{(r)} x_{r e f}\right]
$$

This shows that finding the inverse input $u_{f f}(t)$ is equivalent to finding the reference states $x_{\text {ref. }}$. In other words, asymptotically stable solution for reference state trajectories $x_{r e f}$ is needed. Under state transformation, a portion of the reference states is specified by the desired output and its derivatives in time up to ( $\mathrm{r}-1)$ derivatives. What remains is to find the unknown reference state to determine the forward input.

\section{Augmenting with a Lowpass Filter}

For robustness, the inverse filter should be augmented with a lowpass filter of the form

$$
f(s)=\frac{1}{(\lambda s+1)^{n}}
$$

The order of the filter $\mathrm{n}$ is chosen large to ensure that the augmented inverse system is filter. The variable parameter $\lambda$ determines the speed of response. Small $\lambda$ leads to a small closed loop time constant and high speed of response and vice versa [10]. Horn et als in [11] noted that while the filter in (3) is excellent for tracking and disturbance rejection, its response to load disturbances is poor This is more pronounced in systems with slow dynamics. To overcome this challenge, the researchers proposed a new filter of the form

$$
f(s)=\frac{\beta s+1}{(\lambda s+1)^{n}}
$$

The variable $\lambda$ and $n$ have the same meaning they have in (3). The new variable $\beta$ is chosen to introduce a phase lead in the sensitivity function.

\section{MODELling OF THE FLEXIBLE MANIPULATOR}

The manipulator presented in this paper has 2-flexible links 3D arm. It has a load at the distal end. The manipulator was modeled in MapleSim ${ }^{\mathrm{TM}}$ as shown in Figure 2.

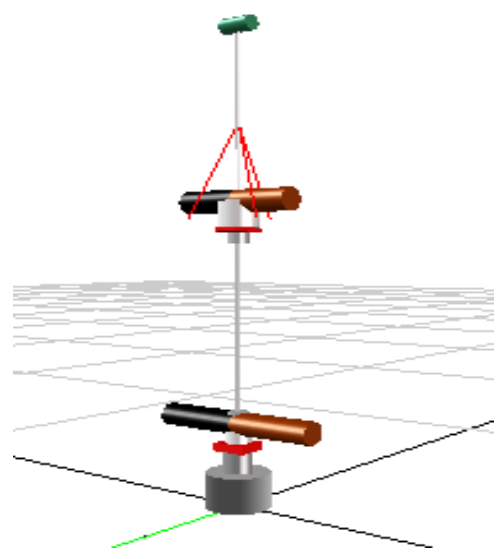

FIGURE II. MANIPULATOR MODEL

The model was linearized to yield the state space model $M(s)$ in Fig 1(a). The same model was used to develop the inverse controller $Q(s)$. Matlab setup of the simulation is shown in Figurer. 3. The three inputs are the reference angles for joint 1,2 and 3 . In the same respect, the nine outputs correspond to the three joint angles, three joint velocities and three strain outputs-torsional, link 1 and link 2.

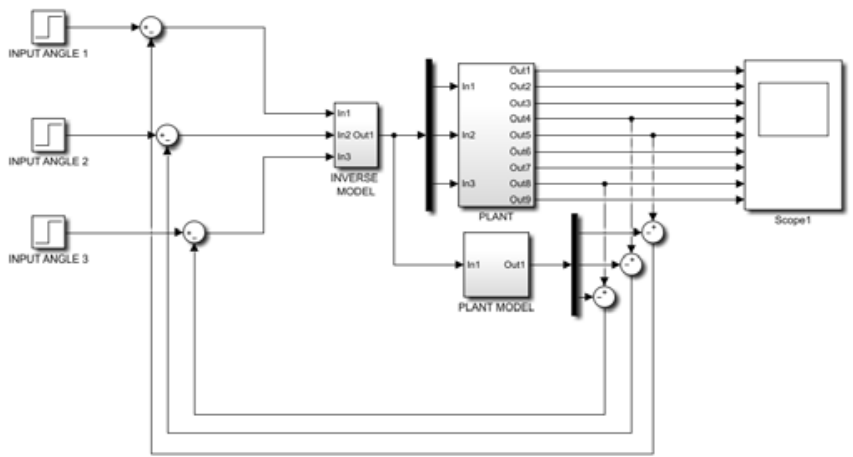

FIGURE III. IMC SIMULATION SETUP

\section{Simulation Results AND Discussion}

The manipulator was excited with a step input corresponding to the desired joint angles. The signal lasted 20 seconds when the all joints were to rotate for 20 degrees and rest for $10 \mathrm{sec}$. After this, the manipulator went back to its vertical position for 10 more seconds.

For $\lambda=0.1$ and $\beta=0.01$, figures 4,5 and 6 show the trajectories tracked by the three joints with and without the internal model controller. The rise time with controller is a bit higher than without controller. 


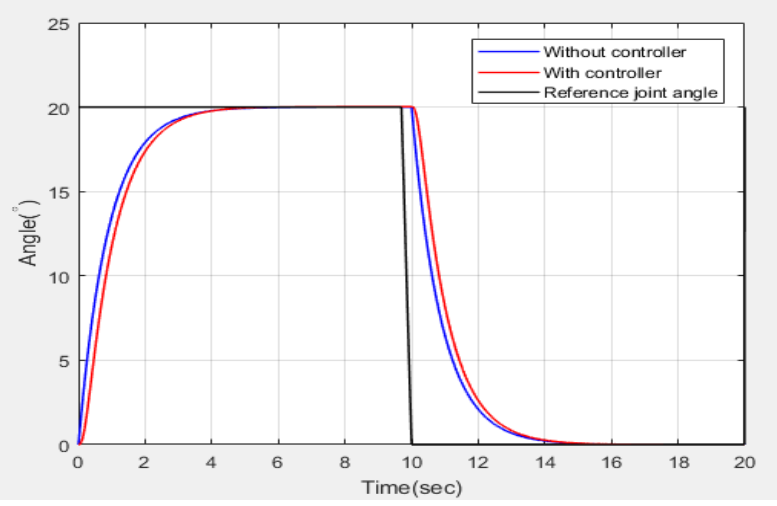

FIGURE IV. POSITION, JOINT 1

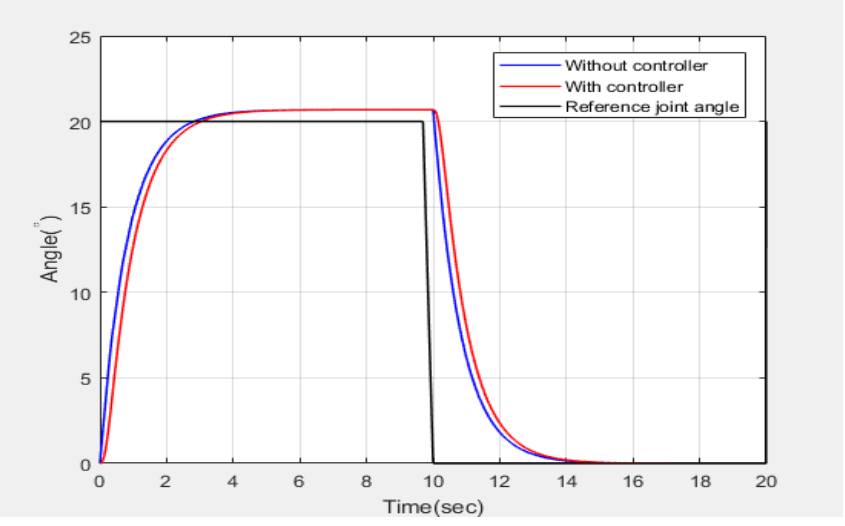

FIGURE V. POSITION, JOINT 2

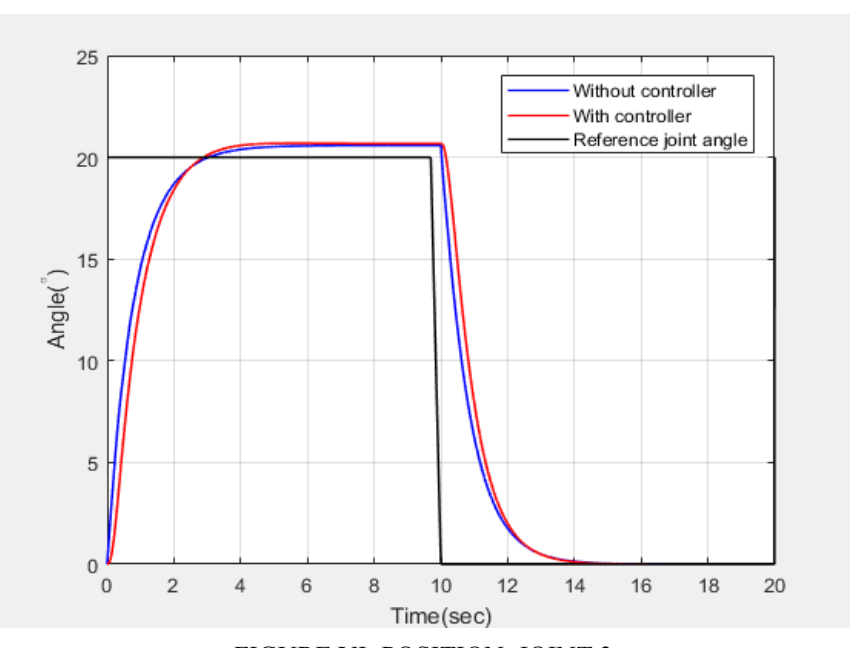

FIGURE VI. POSITION, JOINT 3

Figures 7, 8 and 9 show the strain information. From the figures, there was significant reduction in strain when the internal model controller was used. This is also very evident in the spectral power density of the link strain shown Figures 10, 11 and 12.

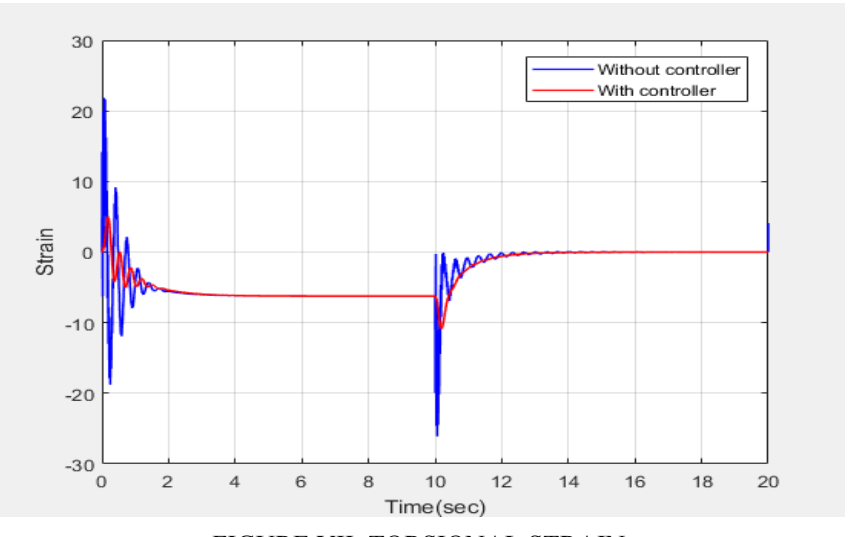

FIGURE VII. TORSIONAL STRAIN

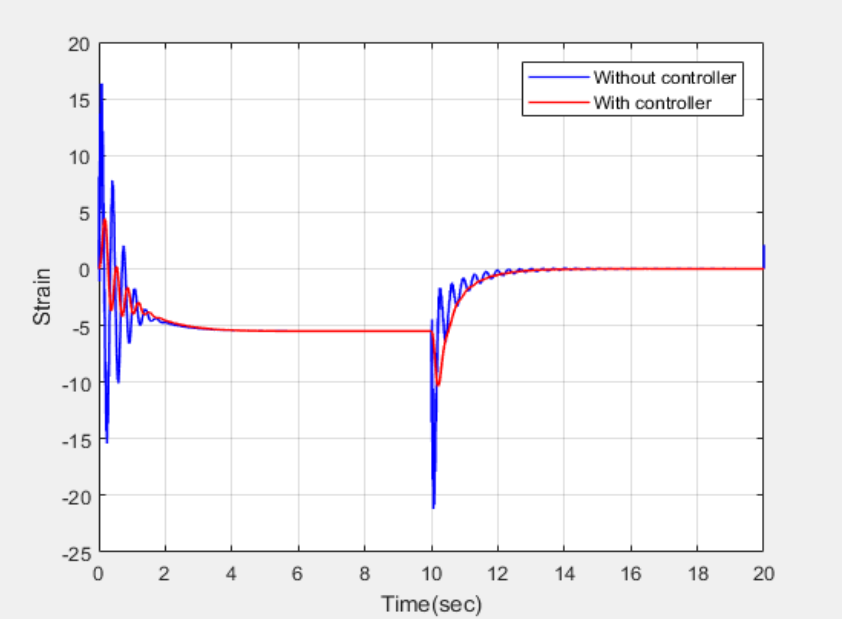

FIGURE VIII. STRAIN, LINK 1

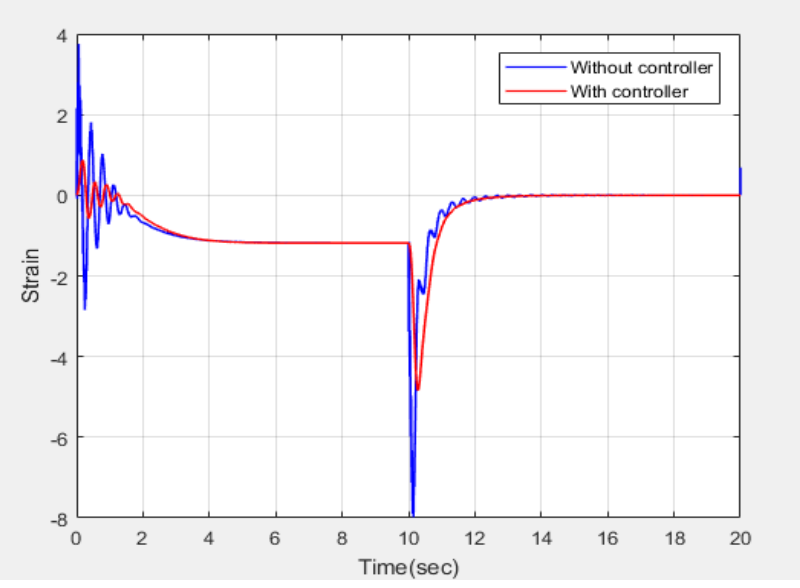

FIGURE IX. STRAIN, LINK 2 


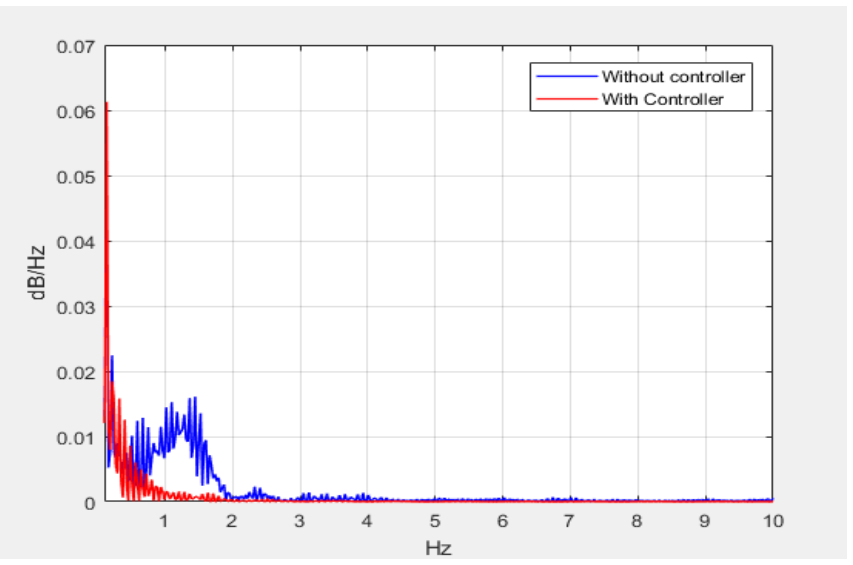

FIGURE X. SPECTRAL POWER DENSITY, TORSIONAL STRAIN

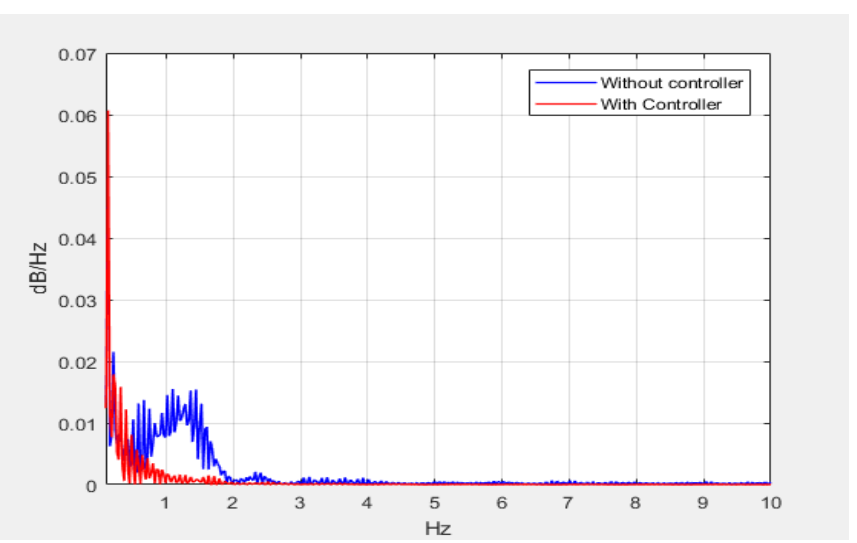

FIGURE XI. SPECTRAL POWER DENSITY, LINK 1

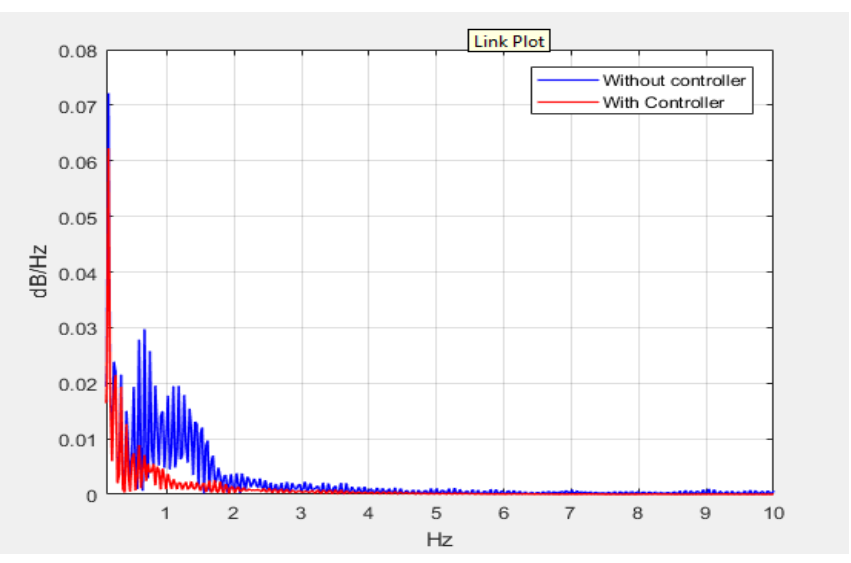

FIGURE XII. SPECTRAL POWER DENSITY, LINK 2

To test for robustness of the IMC system, a step load disturbance equivalent to giving a push for 5 degrees and lasting for $1 \mathrm{sec}$ was introduced to link 2. Figure 13 show the joint position with and without IMC controller. From the figure, the deviation from the reference is significantly reduced in the system with the IMC controller. This is further seen in the strain information in Figure 16 where the ripples are is smooth and doesn't excite more vibrations as can be seen for the system without controller.
Strain spectral power densities for the system with load disturbance are as shown in Figures 15, 16 and 17. Disturbance introduces more vibration modes as is evident by comparing the densities with and without disturbance. However, the IMC system can suppress these additional modes.

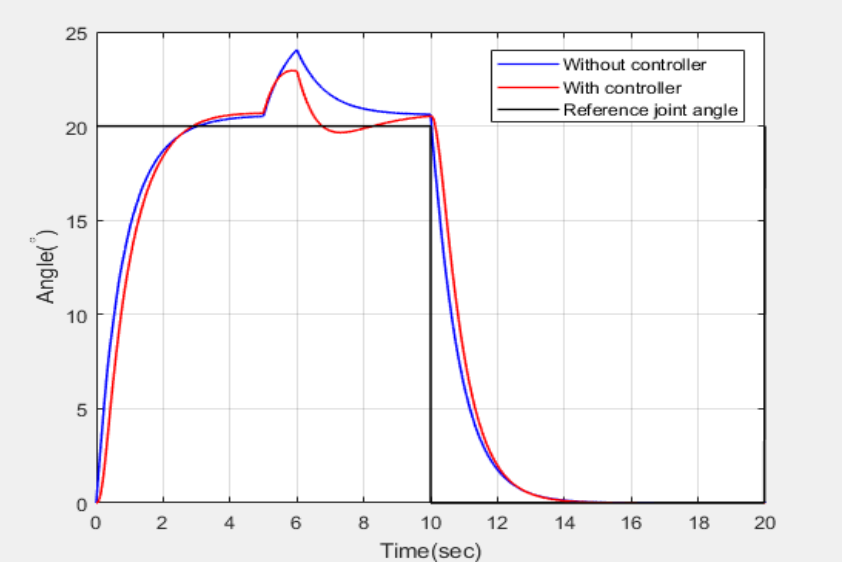

FIGURE XIII. LINK 2 WITH LOAD DISTURBANCE

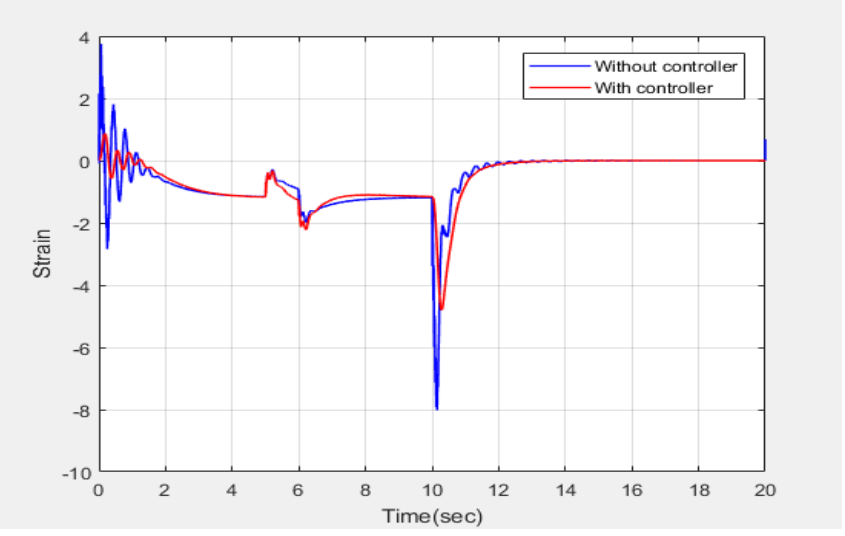

FIGURE XIV. LINK 2 STRAIN WITH DISTURBANCE

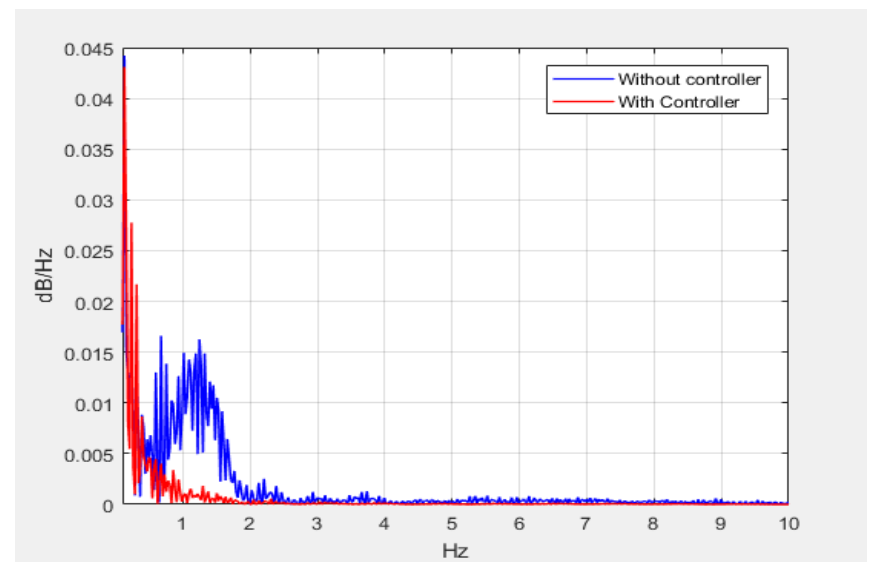

FIGURE XV. SPECTRAL POWER DENSITY, TORSIONAL STRAIN WITH DISTURBANCE 


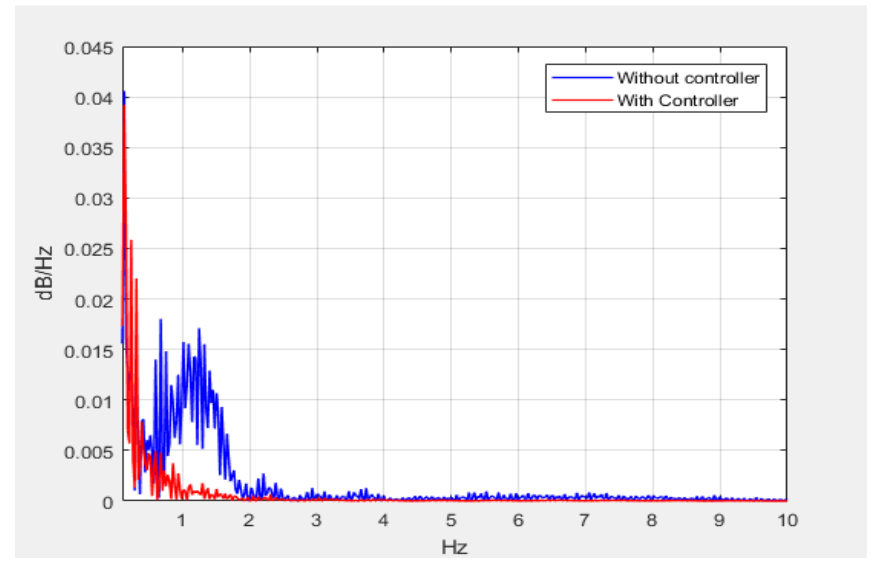

FIGURE XVI. SPECTRAL POWER DENSITY, LINK 1 WITH DISTURBANCE

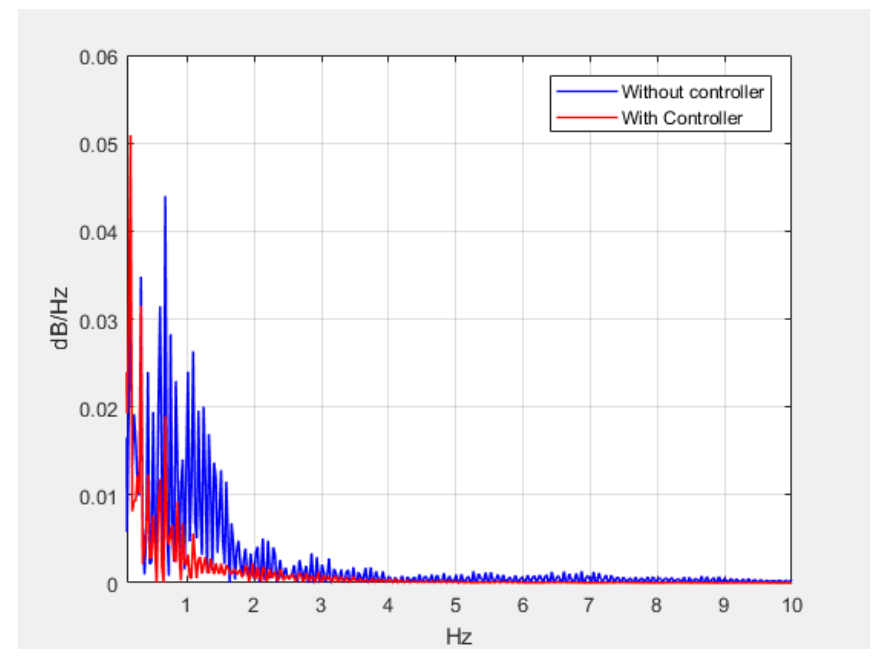

FIGURE XVII. SPECTRAL POWER DENSITY, LINK 2 WITH DISTURBANCE

\section{CONCLUSION}

In this paper, an internal model controlled system system was developed, using a modified filter and applied to control a flexible manipulator. A significant reduction in the link strain is experienced with the application of the IMC system. Also, the system can reject load disturbances.

\section{REFERENCES}

[1] Y. Gao, F.-Y. Wang, and Z.-Q. Xiao, "Chapter 1 - introduction," in Flexible Manipulators, Y. Gao, F.-Y. Wang, and Z.-Q. Xiao, Eds. Boston: Academic Press, 2012, pp. 1 - 3. [Online]. Available: http://www.sciencedirect.com/science/article/pii/B9780123970367000015

[2] Minoru Sasaki, T. Wada, R. Nakamura, and Satoshi Ito, "Vibration control of a flexible manipulator using an adaptive notch filter."

[3] Minoru Sasaki, Kojiro Matsushita, Satoshi Ito, and Takuma Saeki, "Active vibrational control of flexible manipulator using filtered-x lms algorithm," The 2nd DeKUT International Conference on STI \& E, Kenya, 2016.

[4] V. Feliu, K. S. Rattan, and H. B. Brown, "Adaptive control of a singlelink flexible manipulator," IEEE Control Systems Magazine, vol. 10, no. 2, pp. 29-33, Feb 1990.

[5] F. Ghorbel, J. Y. Hung, and M. W. Spong, "Adaptive control of flexible joint manipulators," in Proceedings, 1989 International Conference on Robotics and Automation, May 1989, pp. 1188-1193 vol.2.

[6] R. M. Mahamood, Adaptive Controller Design for Two-Link Flexible Manipulator. Dordrecht: Springer Netherlands, 2014, pp. 115-128. [Online]. Available: http://dx.doi.org/10.1007/978-94-007-6818-5_9

[7] M. Morari and E. Zafiriou, Robust Process Control. Prentice Hall, Englewood Cliffs, 1989.

[8] R. Braatz, "Internal model control," in The Control Handbook. CRC Press Boca Raton, FL, 1996, pp. 215-224.

[9] Zeng Qiu, Mario Santillo, Mrdjan Jankovic, and Jing Sun, "Composite adaptive internal model control and its application to boost pressure control of a turbocharged gasoline engine," IEEE transactions in Control Systems Technology, 2015.

[10] D. E. Rivera, "Internal model control: A comprehensive view," Arizona State University, Tempe, Arizona, pp. 85287-6006, 1999.

[11] I. G. Horn, J. R. Arulandu, and C. J. Gombas, "Improved filter design in internal model control," American Chemical Society, vol. 35, no. 10, pp. 3437-3441, 1996. 